AYANTAGES LT INCONVENIENTS u心s DIVERS SYSTËMES DE TRANSFORMATION

de courant aitariatif à hause tension en courant continu (surTe)

\section{CHAPJTRE XI}

Risques D'ACGIDENo's

Le groupe "synchrone " $\left(^{1}\right)$, la commulatrice et te converlisseur en cascade onl en commun les deux inconvéniculs suivants

$I^{a}$ En cas de compure du circuil d'excilation, l'armature induil dans les inductems des tensions élevées susceptibles de produire des ruptures d'isolant

$2^{\circ} \mathrm{Si}$ l'un des conducteurs du circuit d'alimentation vient i se rompre, la $f$ : e. m. engendrée par le moleur ou la commutatrice s'ajoute à la tension de la ligne el peut meltre cn danger l'isolation de celle-ci. (Pour la commulatrice, l'augmentalion de tension qui se produit de plus au collecteur, en pareil cas, peut même entrainer des coups de feu)

De tels accidents, toutcfois, sont extrêmement rares aucun d'eux, d'ailleurs, no nous a été signalé par les exṕloilants qui onl répondu à notre enquête.

Un danger plus réel du convertisseur en cascade et de la commutalrice est celui qu'ils présentent de pouvoir semballer.

Ponn le convertisseur, si lo disjoncleur à courant allernalif déclam he landis que la nuchine reste connecté du colí contimu, la vilesse monte au double de sa valcur normale II en ast de mème si, le moleur étant accorché, l'excitation vient à manquer du côté conlinu

Pour prévenir loul risque à cel égard, cotte marhine es

(1) Le groupe moteur syuchrone-dynamo. à cause de la rotation de ses inducteurs, est évidemment le plus cxposé aux accidents ponvant provenir du circust d'excrtation. Si cé dernier est alimerté à une tension trop élevée, le fil devient trop fin pour ponvoir se prêter à une construction robuste des électros. Les spires pruvent alors se déplacer sous l'effet do id force centrifuge et, on détermmant l'usure de l'isolant, risquent de provoquer des conrt-circuits locaux à l'mtérien des bobines Ces courtcucuits. dont on ne so rend pas toujours compte assez vite. peuvent-avon des effets très fâcheur pour le moteur. par' suite du martellement des pahers par le rotor qui résulto du céséçulibre magnétique de ce dernier

C"est pourcuol l'excitation du motene par la courant de la dynamo du groupe qu'il actionne n'est pas à recommarder quand il s'agit de tensions supéricures à 500 volts, surtout pour dro puissances infétieúres à 300 kilowatts. (Cette disposition présente d'aillours l'inconvénient, dans le cas de dynamos shunt atilisées sans batteric do fairo diminner l'eventation du moteui upand la charne anumente; co qui est défectucux an double point de vue du facteur de puissarce ot de la stabilité, tout particuliè rement en ce qui conceme les gloupes de traction, que les court-cricuts sur le réseau exposent alor's à de fréquents décrochages)

Voici, à ce sujet, les trusions d'excitation que quelques constructeurs 'm'éconisent de ne pas dépusser' pour toute sécurité

Ateliers de Construetions Electroques du Nard et de l'Fst ; V. max 500 volts

Bchneider et C1e $. V=110$ volts, jusque $50 \mathrm{H} . \mathrm{P} . ; \mathrm{v}=220$ volts andesisus de 500 IIP.

( $\mathrm{v}=125$ volts normalement

British Westinghouse $v=220$ volts entre 500 et $1.000 \mathrm{HP}$ $\mathrm{v} \max =440$ volts all-descris de $1000 \mathrm{JP}$

Cos valeurs maxima so trouvent d'aulleurs généralment observées dans la pratique C'esl ainsi que, panmi les treize installations de groupes synchrones sur lesquelles nous avons été renseignés, denx seulemente comportent l'alimentation de l'excitation des moteurs aux tensions de 550 et 600 volts (respectivement pour des puissances de 250 ou 550 $\mathrm{kw}$ et de $1000 \mathrm{kw}$ ) ; pour toutes les autres, cette alimentation est effectuée ¿े des tonsions de $60 \mathrm{v}, 110 \mathrm{v}$, et $220 \mathrm{v}$ (pour toutes puissances comprises entre 250 et $1.500 \mathrm{kw}$, snit nar le moyen d'une excitatrice colée en bout d'arbre du grnune, soit, quand jl existe une batterre, par le moyen d'une dérivation prise sur une section de celle-ci

Nous n'avons pas trouvé d'exemple d'emploi d'un groupe anxiliaire spécial d'excitation, ce qui s'explique par le grave inconvénient que présente ce dernier de frive dépendre de hii, et nême d'un simple accident à un des circuits inducteurs, la sécurité de tont le service de Ja sous-station toujours pourvue diun limitateur de vilesse à force centrifuge provoquant le déclanchement simultané des disjoncleur's à courant allernatif of à courant comhmu, dès quil se produil un excès de vilesse de $25 " \%$. Par surcroìl de précauhon, on rend de plus ces deus apuareils solidaires, de manière que le déchanchenent de l'un délermine le foncliomement de lautre. Quand celle dernière dispostion n'exste pas, la machine est protégée du côté contrmu au hoyen d'un disjoncleur à relour de courant.

Pour la commulatre simple, I peut y avoir emballenent dun, les differents cas uturanls

a) Lorsquaprès dis jonrhon da colé alternatif, la machine

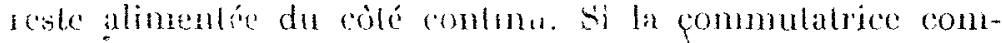

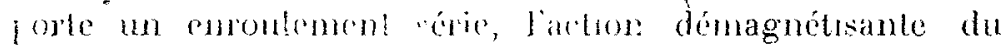

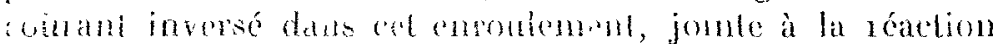
d'induil due an comant magnelsant dis transformateurs,

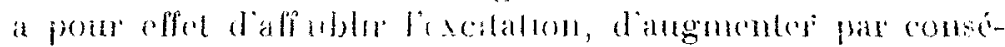

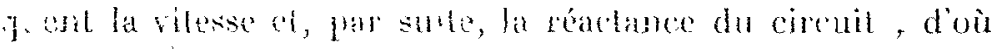

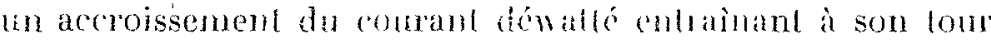
une nouvelle arélélation de vllesene el ainsi de sulle. Cello

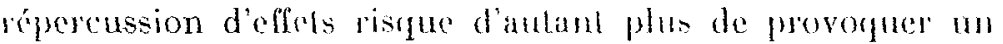
emballement de la commulatrice que celle-ci prescnte une plus grande réartion d'mduil Deux sociétés - les "Tramways de Lille" el les "Tramways de Marseille " nons ont signalé avoir eu le cas d'un pmbilloment survemu dans de s'mblables conditions el ayml abouti, faute dappatcils do prolection ou par suite de défaillance de ces demiers, à l'éclatement do la machine (1).

b) Lorsque la commutatrice est utilisée à rebours pour la transformation du courant continu en courant alternalif, si clle est appeléc à foumir du courant déwalté décalé en arrière, ce dernice, suivant le même processus que ci-dessus, peut aroir pour effet d'entraîner l'emballement.

c) Le même risque existe cncore évidemmenl, Iorsqu'un courl-circuit inductif ayant fait déclancher lo feeder de l'usine centrale, conlinue à être alimenté par la commutalrice.

Pour se garantir contro les conséquences désastreuses do lout amballoment possible, on munit toujours les com-

(1) II nous paraît intéressant de préciser cidessous, suivant les indications que les ducetent's respectifs de ces deux sociétés ont ou l'obligeance de nous domner, les conditions pxinctes duns lesquelles so sont produits les dew accidents.

Tramways de tille - In commutatrice en question. (du typo compound of d'une pussance de $250 \mathrm{kw}$ ). était en selvice dans uno des sousustations du résean (le Breuco) Pour une cause inconnue, le disjonctenr du feeder à hante tension qui l'alimentait vint à déclancher ì l'nsine centrale, bien qu'il fút régré pour une pusssance triple de celle qui correspondait an véglnge du disjoncteur bo conpant continu de la commutatrice, qui était scule en marcho à la sous-station.

Par une mathoureuse concidence la lugne alimentée par cette sous sta. tion se trouva êtie accicmitellement sen contact, à ce moment, avec le fil de trolley d'une ligne déjendant d'un autre résoau (les' Tramways de Rombaiv) Ia commutatrice recevant du courant continu de ce dernier réseau. fonctionna alors on moteur avec son inducteur série inversé, son induit restani commecté au circuit inductif formé par les curoulements secondaires du transformateur , d'oũ. suivant le processus ci-dessus, cmballement progressif jusqu'à l'éclatement

Tramways de Marseille - Ia commutatrice. (d'une puissance de 300 kw ), était en service à li sous-station de la Barasse (fig. 21). Cette sonsstation était alimentée en courant altematif par l'usine a vapenr do St-Giniez qui, par les mémes câbles. foumissait du courant au poste de trausformation d'Allauch.

Ia sous-station de la Barasse assurat son service normal et distribuait du conrant contina sur le résean de Tramways Elle était, d'antre part, en parallèle du côté continu nar lintermédiaire des fils do trolley avec une autre des sous-stations da résea a (Castellane).

A un moment donné. "Tssine de St-Giniez coupa la' haute tension" sans inertir la sous-station de la Rarasse. La commutatrico de cette dernièro sous-station recevant ainsi du courant continu de la sous-station do Castellane, envoya du comant alternatif at poste d'Allauch qui se treuvait relié à des apoareils possédant une unande self-induction. Il y ent alors un fort appel de coupant déwatté qui démagnétisa les inducteurs de la commutatrice et détermina, son emballement. 
mulatrices travaillant à rebours d'un limitateur de vitesse commandant le disjoncteur à courant continu ; dans tous les autres cas, on se contente généralement d'adjoindre à ce dernier disjoncleur, un relai à retour de courani empèchant la machine de travailler comme moteur.

Les commutalrices présentent encore un autre inconvénient qui leur est partıculicr. Elles sont sujettes à des inversions de polaritć qui peuvent survenir en service, soit à la suite d'ume forte baisse de tension du côté alternatif, soil après une grande surcharge du côté continu. De telles inversions se sont produites dans un certain nombre d'installations ayant fail l'objel des réponses que nous avons recues, mais elles, n'ont pas eu, gràce au fonclionnement des disjoncleurs, d'autre conséquence fâcheuse que l'interruption occasionnéc pendant le temps nécessarre au rétablissement de la bonne polarité

Le moterir d'induction, d'autre part, n'offre évidemment aucun des inconvénients qui précèdent. Par contre, quana i) est alimenté directement à hauto tension, il se trouro exposé de son còté aux accidents suivants :

lor's de sa mise cn circuit, par surte de la réllexion de l'onde de-tension, la self-inducion de l'enroulement peut faire supporter aux premières spires la tolalité de la tension de service, d'où des risques de claquage ${ }^{1}$ ). On évite ce danger en plaçant aux bornes du stator une résistance amorlissant le front d'onde ou, mieux, une self faisant oflice de bobine de choc.

Il peut se produire aussi, lors de la mise sous tension brusque, surtont dans le cas d'un moteur fórtement saturé ct à faible entrefer, des surintensités considérables ('z). Ces ¿̀-coups d'intensité n'ont, en général, pour le moteur, d'autre effet que de le soumettre à de fortes vibrations ; toutefois, à la longue, ils peuvent fatiguer les isolants; ils ont aussi quelquefois pour conséquence d'introduire des troubles dans le réseau par l'amorçage des disposilifs de protection. Une résistance en série avec le motcur permet encore d'obvier à ces invonvénients, on obtient en général unc'réduction suffisante do l'intensilé el de la durée du courent de fermeture en maintonant seulcment en circuit. pendant quelques centièmes de seconde, une résistance capable d'absorber ro à $15 \%$ de la tension du réseau. Cette résistance est le plus souvent placée entre contacts princifaux et plots auxiliaires à l'intérieur de l'interrupteur $\left(^{(3)}\right.$.

Le converlisscur en cascade et les transformateurs statiques sont également soumis évidenment, aux deux effets précédents. Mais pour les transformaleurs, il esl aisé de prévoị les premières bobines avec un isolement suffisamment l'cnforcé pour leur permettre de supportes en loute sécurité le choc de la lension tolale. Ouant aux surintensjés. clles n'ont pas d'inconvénients graves pour ces appareils, si les bobines sont mécaniquement très bien assujetties.

D'un autre côté, on peut observer que le moteur asynchrone; en raison de la très faible valeur de son entrefer, se trouve plus exposé que les autres machines aux accidents pouvant provenir d'un échauffement anormal des paljers. Pour les très haules lensions, il faut comptor aussi avec le

(1) Pareil accident s'est produit notamment dans les installations de la ville cle Glasgow et de "The London County Council "sur des moteurs de groupes de $1.000 \mathrm{kw}$. et $500 \mathrm{kw}$ alimentés à la tension de 6600

(2) On sait en effet que. si la mise en circuit a lieu dans le voisinage du zéro de la courbe de tension, le flux magnétique atteint dans la première période à peú près le double de la valeur normale.

(3) Quand les groupes asynchínnes sont installés dáns une usine centrale comportant un système de mise en chäge progressive de feeders. leurs moteurs peuvent être mis graduellement sous lension comme des feeders et les causes d'accident ci-dessus se trouvent ainsi radicalement
sunprimées danger des décharges électriques possibles entre rotor et stator. Cel inconvénient limite dans une certaine mesure l'emploi des lensions élcvées pour les moleurs asynchrones. Mais la lension de ces moleur's se trouve encore plus étroitement limitée par les difficultés qu’il y a de réaliser un très bon isolement offrant toute sécurité entre les nombreux Cils qui doivent passer dans une même encoche. En fait, f'accroissement du prix et la diminution du rendement cntraînés par l'augmentation de la lension font qu'on ne dépasse pas le plus souvent les valeurs (4) ci-dessous indiquées :

$\begin{array}{rl}\mathrm{P}<150 \mathrm{kw} . & \mathrm{V}_{0}=3.000 \mathrm{~V} \\ 150 \mathrm{kw}<\mathrm{P}<300 \mathrm{kw} . & \mathrm{V} .=5.000 \mathrm{~V} \\ 350 \mathrm{kw}<\mathrm{P}<1.000 \mathrm{kw} . & \mathrm{V} .=6.600 \mathrm{~V} . \\ 1000 \mathrm{kw},<\mathrm{P} & \mathrm{V} .=10.000 \text { à } 22.000 \mathrm{~V} .\end{array}$

Pour le moteur synchrone, les seules difficultés qui s'opposent à l'emploi de tensions trop élevées sont celles qui ont trait à J'isolation; cos difficultés sont évidemment beaucoup moindres que pour lo moleur précédent, au moins pour les bobines d'entrée ; on ne dépasse pas néanmoins, par mesure de sécurité, la tensíon de 12.000 volts.

D'autre part, en ce qui concerne la commutatrice, les transformateurs statiques dont elle exige l'emploi comportent à la fois un avanlage et un inconvénienl. En mellant la haute tonsion hors de la portée du personnel chargé dc la conduile des machines, ils soustraient ce dernier à unc source de danger. Mais, d'un autre côté, la grande quantité d'huile qu'ils renferment constitue pour, l'installation, au point de vue des risques d'incendie, un élément très dangereux. Tout péril grave peut cependant être évité à cc sujel si, conformément à la pratique qui tend à se généraliser de plus en plus, on place les transformateurs en dehors de la sous-station, dans des loges complètcment séparées les uncs des autres, et si on prévoit des canivaux permettant l'évacuation rapide de l'huile en cas dc nécessité.

Enfin, iI est bien évident qu'au point de vue des risques d'accidents pouvant résultor de fausses manœuvres, dnes soit à la négligence, soit à l'incompélence du personncl, lo groupe motcur-asynchronc-dynamo et le convertisseur en cascade sont les deux groupes qui présentent la plus grande sécurité. Ces risques, toutefois, sont très minimes avec de bons appareils de protection.

\section{CHAPITRE XII}

\section{Observations DIVERSES}

I - Marche en parallèle de groupe de système différents.

L'utilisation en parallèle de groupe moleur-générateurs "synchrone" et "asynchrone ", ne présente naturellcment pas de difficulté, la vitesse des deux espèces de moteurs obéissant sensiblement de la même manière aux variations de la fréquence, et les variations éventuelles de la tension alternative restant sans effet sur la tension continue.

La commutatrice, par contre, ne peut, être utiliséc concurremment avec ces groupes rue si elle comporte un survolteur ou un enroulement série (1) avec self additionnolle ; fencore, le fonctionnement reste-t-il très délical

(1) Ces tensions peuvent être tointefois dépasséeg quand des circonstances particulières l'exigent. Ainsi on peut, ì la rigueur, construire dés moteurs de $200 \mathrm{~K}$. W. pour une ténsion de 10.000 volts. mais le prix de tel Is moteurs devient très élevé quand on veut réaliser dans lem construction, au point de vue de l'isolation, toutes les conditions requises pour une parfaite sécurité de service

(2) Des commutatrices à cnrovlement série discordant: sont ainsi nor malement utilisées en parallèle avec des groupes moteurs-génératenrs, à l'usine d'électricité de la ville de-Glasgow sans' qu'aucun inconvénient, autre oue les variations dn $\cos \varphi$ dues aux variations de charge, se soit. manifestá 
‘uns ces conditions, l'égale réparation des charges entre machincs ne pouvant se maintenir que si la tension alter: ative ne varie pas.

Quant au converlisseur en cascade, sa mise en parallèle arec un groupe moteur-générateur peut être réalisée aisément sans autre artifice que le déplacement, par rapport au milieu de l'espace interpolaje, des lignes de balais et des épanouissements des pôles de commutation (1), la réaction d'armalure ainsı créée suffisant à produire la varialion do unsion voulue. A défaut de pôles auxiliaires, une excitation hypocompound est toutefois nécessaire. Dans les deux cas, lo partage exact des charges entre groupes reste, comme précédemment, essentiellement dépendant de la stabilité de la lension alternative.

\section{II. - Marche en parallèle avec une batterie-tampon.}

Les groupes moteurs-générateurs offrent évidemment l'avantage de pouvoir' travailler directement en parallèle avec une batleric sans qu'aucun dispositif spécial soit nécessaire.

La commulatrice et le convertisseur en cascade exigent, pour pouvoir être ulilisés dans les mêmes conditions, l'emploi d'une des dispositions ci-dessus indiquées. Toutefois, la batterie ne peut alor's remplir convenablement son office régulateur que si la tension alternative d'alimentalion n'est influencée que par la charge des machines en parallèle avec elle. Dans la négative, les variations de la tension alternalive dues à toule aulre carıse ont pour effel de rendre loul à fail arbitraire la répartition de la chargr du réseau cntre. les machines et la balterie.

Mais il esl rare, en pratique, du moins en malière de traclion, qu'une batlerie-tampon soil utilisée, ainsi que nous venons de l'envisager, en connection directe avec les machines dont clle doit régler le régime. Il y a, cn cffet, à l'emploi d'une telle disposition, dewx sortes d'inconvénients D'une part, à cause de la chute de lension dur à la résislance intérioure de la batterie, il existe un ćart asser considérable entre la tension au-dessus do laquejle la batterie peul se charger et colle au-dessous dr laquelle elle se décharge, de sork que la ballerietampon laisse ainsi subsister aux barres des machines, des valuations de tension (de l'ordre de ro \%), généralcuenl inadmissibles. D'autre part, la batterie ne peut interunis offeranment dans l'alimentalion des à-coups que si $:$ l ésistance intérieure esl très faible, ot celte condition, oblige à cmployer des éléments d'une capacité boaucoup plus grande que celle qui rorrespondrait au débit nécessaire.

C'est pourfuoi l'utilisation rationnclle d'une batterielampon doil toujours rominler, même avec les groupes noleurs-générateurs, f'amploi d'un survolteur dévolteur automatique, ('n série aver la balteric. Dans ces conditions, toulc la différence qui cxisle mitre ces groupes ot les deux autres catégories de machines (commulatrice et 'onverlisseur en cascade), au point de vue qui nous occupe, se réduit à ce que ces dernières nécessitenl, au lieu d'un simple survoltetar série, un survoltcur différentiel $\left({ }^{2}\right)$, à deux

(1) C'est ainsi que pour des convertisseur's en cascade destinés à fonctromer en parallelc avec des moteurs-générateurs à forte chute de tension, (spécialement réalisée pour limiter l'intensité des courts-circuits 'ventuels du réseau). on a pu obtenir par ce moyen, à i’usine précitée, des chutes de tension de 18 à $20 \%$, la chute correspondant à la réaction naturelle de ces machines n'étant que de $5 \%$ environ.

(2) Iro principe de l'action du survolteur dufférentiel consiste, comme nn sait. en ce que toute variation du courant des machines, quelle qu'en soit la cause, détermine dans ce survolteur, et par conséquent dans 1 c circuit de la batterie, la production d'uue f e.m. dont l'effet est de s'oppo. ser à cette variation ck courant Celle-ci cst d'autant plos rédurte que l'en

roulement série du survoltenr parcouru par le courant des machines est ou trois enroulements, dont le seul inconvénient par rapport au précédent est de consommer en pertes "Joule " un peu plus d'énergie, par. suite de ce que les ampères-tours de l'cnroulement série-machine correspondant au courant moyen doivent être constamment équilibrés par les ampères-tour's de l'inducteur à fil fin. Mais ce survolteur différenticl est évidemment aussi indispensable avec les moteurs-génératcurs quand leur-génératrice est compound ou hypercompound, de sorte que tous les systèmes deviennent alors, au point de vue considéré, absolument équivalents.

\section{III. - Invcrsibilité.}

L'occasion d'effectuer la Iransformation inverse de courant continu en courant alternatif se présente parfois dans les sous-stations de traction, pourvues d'une batterie-tampon et utilisées comme centres de distribution des feeders à haute tension, d'un réseau d'éclairage desservi par la même usinể contrale.

En pareil cas, les groupes de transformation ulilisés à rebours, après l'arrèt du service de traction, peurent aljmenter, au moyen de l'énergic emmagasince dans la batterie, la faible charge requise pendant la nuit par le résean d'éclairage, ce qui permet d'arrètor éventuellement leś machines de l'usine.

Le groupe moteur-asynchrone-dynamo est évidemment incapable d'opérer celte transformation inverse. Au contraire, le groupe moteur-synchrone-dynamo est, de tous les systèmes, le mieux approprié à sa réalisation, car il permel d'engendrer le courant alternatif à une fréquence constanle el sous une tension aisément réglable à volonté. Cette ćgale aptilude à réaliser l'une ou l'autre transformation est une cualilé très précieuse du groupe "synchronc ", de nalıle à justifier son choix toutes les fois que la nócessité de transformer du courant continu en courant alternatif doit se présenter fréquemment d'une façon normale.

la commutatrice et le convertisseur rn cascade ne se prêtenl que d'une manière imparfaile à l'accomplissement da la lransformalion considéréc.

Pour la commulatrice, la fréfuence du courant altcrnalif débilé dépend;" en offet, de la valeu de la composante déwaltéc de ce dernier ; elle est done essenticllement ins lable, à noins que celte machine ne fonctionne on parallèle avec une autre souree de courant alternatif de fréquence constantc (auquel cas cette dernière source supporte toutes les variations du courant déwalté absorbé par le réseau). D'autre part, le volfage diminue quand l'intensité ou le retard du courant augmentent of on ne dispose. d'aucun moyen pour le régler.

Les mêmes inconvéuients existent pour lo corvertisseur cn cascade, avec cette différence que l'eflet des courants déwatlés sur la fréquence est beaucoup moindre que pour la tension provoguées par ces courants sont considérablement 1)lus accentuées que pour celte dernière machine, à cause de la plus grande réaclance en jeu.

\section{T. Sanrat, \\ Ingénieur à la Co Ge de Railways el d'Eledricilé de Bruxelles.}

plus important. A cet égard, le survolteur pourvu de deux curoulement, indépendants. (série machine et série batterie) est plus souple pt plus eff cace que le survolteur dans lequel ces deux inducteurs sont confondus dans un seul enroulement série-feedlers, car le réglage de ce dernier résul tant de la valeur de la résistance de la batterie, on ne peut pas disposon librement de sa puissance.

On pout supprimer la légère variation du, courant des machines que laisse subsister lo survolteur en faisant agir sur l'inducteur en dérivation pris aux bornes de la batterie. un régulateur automatique à déclic. L'inducteur série ne laísse alors à ce régulateur dont la course se trouve réduite, qu'un travail d'ajustage.: (cette disposition a été notamment réalisée à la sons-station de la ligne de Porte-Ceresio à Milani. 
TABLEAU XVI. - c) Résumé des réponses reçues concernant les Sous-Stations

\begin{tabular}{|c|c|c|c|c|c|c|c|c|c|c|c|}
\hline \multirow{3}{*}{ RÉSEAUX } & \multicolumn{4}{|c|}{ USINE GENEIATRHL } & \multirow{3}{*}{ Services } & \multirow{3}{*}{ 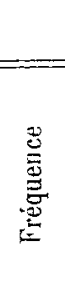 } & \multirow{3}{*}{$\begin{array}{l}\text { Haute tension } \\
\text { alterildive } \\
\frac{10}{10} \text { ts }\end{array}$} & \multirow{3}{*}{$\begin{array}{c}\text { Tension } \\
\text { continue } \\
\text { volts. }\end{array}$} & & & \\
\hline & \multirow{2}{*}{$\begin{array}{c}\text { Puissance totale } \\
\text { n stallée } \\
\text { KII }\end{array}$} & \multirow{2}{*}{ Services réalısis } & \multirow{2}{*}{$\begin{array}{l}\text { Varmation } \\
\text { de frrífuence } \\
\text { en servce }\end{array}$} & \multirow[b]{2}{*}{$\operatorname{Cos} \varphi$ mujel } & & & & & \multicolumn{3}{|c|}{$\begin{array}{c}\text { Conditions } \\
\text { d'explutation moyennes } \\
\text { sous to } \\
\end{array}$} \\
\hline & & & & & & & & & 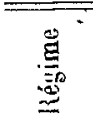 & 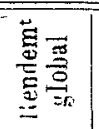 & 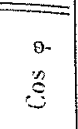 \\
\hline \multirow{3}{*}{$\begin{array}{c}\text { Tramways } \\
\text { municipaux de } \\
\text { Saint-Gall }\end{array}$} & \multirow{3}{*}{$\begin{array}{l}\mathrm{MV}, \mathrm{TV} \text { et TH } \\
\text { de } 200 \mathrm{HP} \\
\grave{a} 1500 \mathrm{HP} \\
\Sigma=4600 \mathrm{HP}\end{array}$} & \multirow{3}{*}{$\begin{array}{l}\text { Charge moyenne } \\
1560 \text { HP dont : } \\
\text { Traction .. } \\
\text { Eclairage. } \\
\text { F. motrice. } \\
1000\end{array}$} & \multirow[t]{3}{*}{$\begin{array}{l}\text { Négligeable } \\
\text { chaige fixe }\end{array}$} & \multirow{3}{*}{$\begin{array}{c}\text { Mloyenne } \\
\dot{0}, 7 \\
\text { varie de } \\
0,65 \\
\text { à } \\
0,80\end{array}$} & \multirow[t]{3}{*}{$\begin{array}{l}\text { Traction } \\
\text { et } \\
\text { Eclaurige }\end{array}$} & \multirow[t]{3}{*}{50} & \multirow[t]{3}{*}{$\begin{array}{l}3550 \\
\dot{d} \\
3650\end{array}$} & \multirow{3}{*}{$\begin{array}{c}\text { Triction } \\
600 \\
\text { Eclars et Force } \\
250\end{array}$} & $\begin{array}{c}0,70 \\
i \\
1 / 1\end{array}$ & $\begin{array}{l}\text { ens. } \\
0,80\end{array}$ & $\begin{array}{l}0,65 \\
\dot{a} \\
0,80\end{array}$ \\
\hline & & & & & & & & & Grou & de 17 & $k w$ \\
\hline & & & & & & & & & ${ }^{b_{i}}{ }_{i k}$ & 0,85 & 0,90 \\
\hline \multirow{5}{*}{$\begin{array}{c}\text { Centrale } \\
\text { électrique de la } \\
\text { Ville } \\
\text { de Liverpool }\end{array}$} & \multirow{5}{*}{ 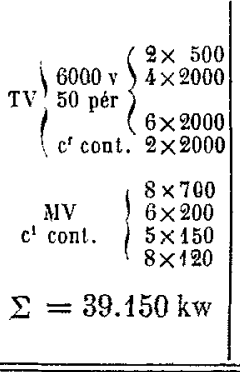 } & \multirow{5}{*}{$\begin{array}{c}\text { Charge moyenne } \\
\text { en hiver : } \\
\text { Traction } 5000 \mathrm{kw} \\
\text { Eclairage et Force } \\
\text { motrice } 5700 \mathrm{kw}\end{array}$} & \multirow{5}{*}{$<1 \%$} & \multirow{5}{*}{$\begin{array}{c}0,71 \\
\text { à } \\
0,89\end{array}$} & \multirow{5}{*}{$\begin{array}{l}\text { Tracisou } \\
\text { et } \\
\text { Eclairage }\end{array}$} & \multirow[t]{5}{*}{50} & \multirow[t]{5}{*}{6000} & \multirow{5}{*}{$\begin{array}{l}\text { Traction } \\
\qquad 540 \\
\text { Eclairage } \\
480\end{array}$} & ) & D & $"$ \\
\hline & & & & & & & & & \multicolumn{3}{|c|}{ Groupe de $200 \mathrm{kw}$} \\
\hline & & & & & & & & & $\begin{array}{l}4 / 4 \\
2 / 4\end{array}$ & $\begin{array}{l}0,845 \\
0,81\end{array}$ & $\begin{array}{l}0,91 \\
0,83\end{array}$ \\
\hline & & & & & & & & & Grou & e de 50 & $0 \dot{\mathrm{k} w}$ \\
\hline & & & & & & & & & $\begin{array}{l}5 / 4 \\
2 / 4\end{array}$ & $\begin{array}{l}0,86 \\
0,83\end{array}$ & $\begin{array}{l}0,89 \\
0,80\end{array}$ \\
\hline $\begin{array}{c}\text { Chomin de fer } \\
\text { Montreux- } \\
\text { Oberland } \\
\text { Bernois }\end{array}$ & $\mathrm{MV}=6000 \mathrm{HP}$ & D & 》 & $\eta$ & $\begin{array}{c}\text { Traction } \\
\text { exclusivement }\end{array}$ & 50 & $\begin{array}{c}8.000 \\
\dot{\mathrm{a}} \\
8.800\end{array}$ & 800 & 0,70 & 0,82 & 0,87 \\
\hline
\end{tabular}

TABLEAU XVI. - d) Résumé des réponses reçues concernant les

\begin{tabular}{|c|c|c|c|c|c|c|c|c|c|c|c|}
\hline $\begin{array}{c}\text { Tramways } \\
\text { electriques de } \\
\text { Buda-Post }\end{array}$ & $\begin{array}{l}\mathrm{TV}\left\{\begin{array}{l}2 \times 1635 \\
2 \times 4000\end{array}\right. \\
\Sigma=11.270 \mathrm{kw}\end{array}$ & $\begin{array}{c}\text { Traction } \\
\text { exclusivement } \\
\text { P moy. } 3500 \mathrm{kw} \\
\text { Variations } \\
\text { de } 1100 \mathrm{kw}\end{array}$ & Négligeable & 1 & $\begin{array}{c}\text { Traction } \\
\text { exclusivement }\end{array}$ & 50 & $\begin{array}{r}10.000 \\
+\quad 2 \%\end{array}$ & 550 & $3 / 4$ a $4 / 4$ & 0,86 & 1 \\
\hline $\begin{array}{c}\text { Tramways } \\
\text { municipaux de } \\
\text { Vienne }\end{array}$ & $\begin{array}{l}\mathrm{MV}=10 \times 2000 \\
\mathrm{TV}\left\{\begin{array}{r}1 \times 350 \\
6 \times 6000 \\
2 \times 7200\end{array}\right. \\
\Sigma=70.750 \mathrm{kw}\end{array}$ & $\begin{array}{c}\text { P moy. } 18040 \mathrm{kw} \\
\text { dont : } \\
\text { Traction. } 6550 \mathrm{kw} \\
\text { Eclairage } 5370 \mathrm{kw} \\
\text { F. motrice } 4800 \mathrm{kw} \\
\text { Sources } \\
\text { de l'exploitation } \\
\text { méme : } 1320 \mathrm{kw}\end{array}$ & $\begin{array}{l}\text { Négligeable } \\
\text { la charge } \\
\text { ne subissant } \\
\text { pas dé } \\
\text { variations } \\
\text { brusques }\end{array}$ & $\begin{array}{c}0,98 \\
\grave{a} \\
1\end{array}$ & $\begin{array}{l}\text { Traction } \\
\text { et } \\
\text { Eclairage }\end{array}$ & 48 & $\begin{array}{c}5: 000 \\
\text { à peu près fixe } \\
\text { la charge } \\
\text { variant très peu } \\
\end{array}$ & $\begin{array}{c}600 \\
\text { pour traction } \\
\text { et } 450 \\
\text { póur éclairage }\end{array}$ & $\begin{array}{l}4 / 4 \\
2 / 4\end{array}$ & $\begin{array}{l}0.884 \\
0,842\end{array}$ & 1 \\
\hline \multirow{4}{*}{$\begin{array}{c}\text { Tramway } \\
\text { et Société } \\
\text { d'Electricité de } \\
\text { Linz-Urfahr }\end{array}$} & \multirow{4}{*}{$\begin{array}{l}\text { Enorgie achelée } \\
\text { à une Centrale } \\
\text { hydraulique } \\
\text { étrangère. } \\
\text { Usine à vapeur } \\
\text { de secours } \\
\text { de } 2000 \mathrm{kw}\end{array}$} & \multirow{4}{*}{$\begin{array}{l}\text { Charg: moyenne : } \\
\text { Traction. } 500 \mathrm{kw} \\
\text { Eclarrage } \\
\text { et Force. } 1500 \mathrm{kw} \\
\text { Tension : } \\
25.000 \mathrm{~V} \text { pour } \\
\text { transport à grande } \\
\text { distance } \\
2000 \mathrm{~V} \text { pour } \\
\text { la Ville }\end{array}$} & \multirow[t]{4}{*}{$D$} & \multirow[t]{4}{*}{$"$} & \multirow{4}{*}{$\begin{array}{c}\text { Traction } \\
\text { et moteurs } \\
\text { branchés } \\
\text { directement } \\
\text { sur le réseau } \\
\text { de traction }\end{array}$} & \multirow[t]{4}{*}{50} & \multirow[t]{4}{*}{2.000} & \multirow{4}{*}{$\begin{array}{c}580 \\
\grave{a} \\
600\end{array}$} & \multicolumn{3}{|c|}{ Groupe de $110 \mathrm{kw}$} \\
\hline & & & & & & & & & 0,75 & 0,75 & $\begin{array}{l}0,80 \\
3 \\
0,90\end{array}$ \\
\hline & & & & & & & & & \multicolumn{3}{|c|}{ Groupe de $400 \mathrm{kw}$} \\
\hline & & & & & & & & & 0,75 & 0,86 & \\
\hline
\end{tabular}


équipées exclusivement au moyen de groupes " moteur asynchrone-dynamos" (StITE).

SOUS-STATIONS DE TRANSFORMATION

\begin{tabular}{|c|c|c|}
\hline $\begin{array}{l}\text { Nature et Puissance (en Kw) } \\
\text { des } \\
\text { Groupes de Transformation installés }\end{array}$ & $\begin{array}{c}\text { Puissance } \\
\text { totale } \\
1 \quad \text { jo... } \\
\mathrm{Kw}\end{array}$ & $\begin{array}{l}\text { I. Observations relatives à la justıfication des systèmes employés. } \\
\text { II. Observations diverses. }\end{array}$ \\
\hline $\begin{array}{l}\text { Moteur entrainant deux dynamos shunt } \\
\text { (a pules auxiliaires) couplées en purallèle } \\
\text { quand elles travallent sur le réseau d'éclar- } \\
\text { rage et en sérı quand eiles alimentent le } \\
\text { réseau de traction. } \\
3 \text { groupes de } 50 \text { i } 500 \mathrm{HP} \ldots \ldots \ldots \ldots \text {. . . . }\end{array}$ & $\cos 500$ & 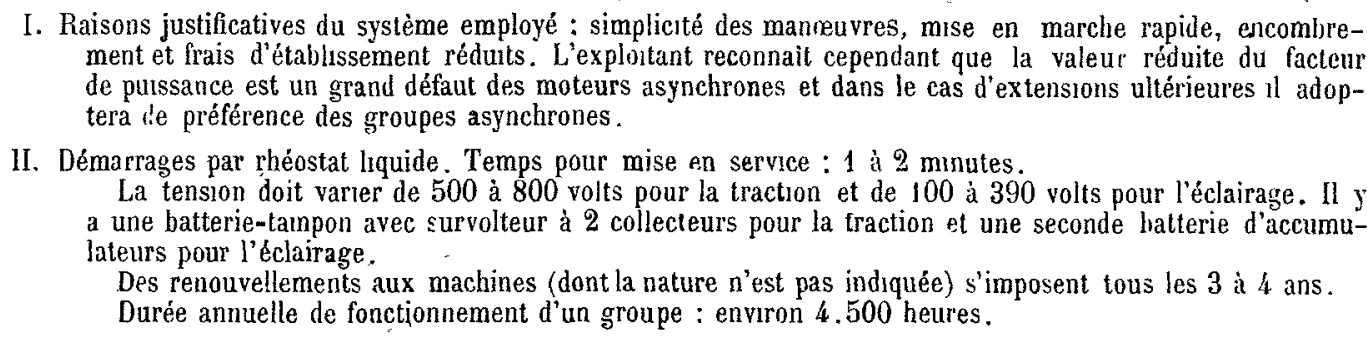 \\
\hline $\begin{array}{l}\text { Moteur dyuamo shunt } \\
6 \text { groupes de } 1000 \mathrm{kw} \text { alimentés à } 330 \mathrm{~V} \\
\left.\begin{array}{l}33 \text { groupes de } 200 \mathrm{kw} \\
29 \quad 500 \mathrm{kw}\end{array}\right\} \text { alımentés à } 6000 \mathrm{~V}\end{array}$ & 21.700 & 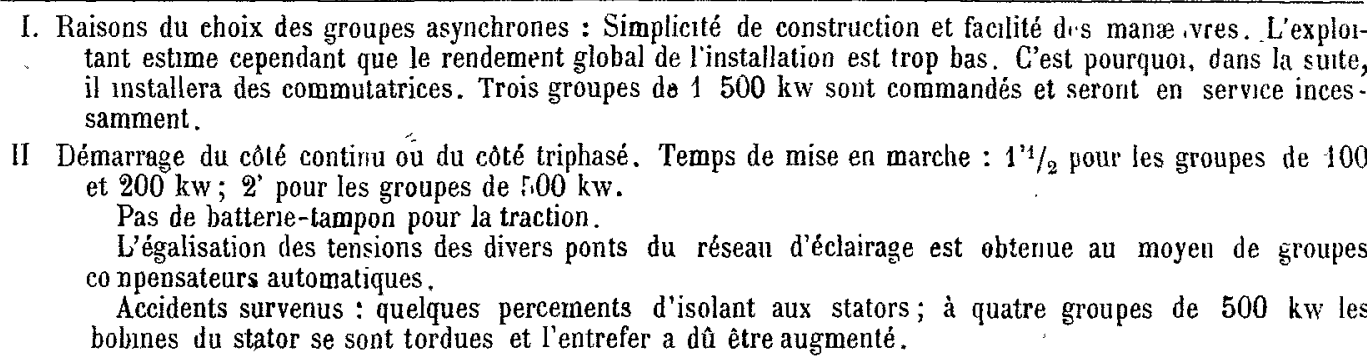 \\
\hline $\begin{array}{l}\text { Moteur-dynamo shunt }(1904.07) \\
1 / 4 \text { groupes de } 180 \mathrm{kw} . \ldots \ldots \ldots . . .\end{array}$ & 2.520 & $\begin{array}{l}\text { I. Groupes asynchrones choisis à cause de leur faclité de démarrage. } \\
\text { II. Démarrage par rhéostat liquide. Temps de mise en marche environ l'. } \\
\text { Les groupes travaillent en parallèle avec une batterie-tampon sans survolteur. } \\
\text { Accidents survenus : claquage d'isolant à la mise sous tension brusque du stator. } \\
\text { Durée annuelle de fonctionnement d'un groupe : environ } 4.500 \text { heures. }\end{array}$ \\
\hline
\end{tabular}

\section{Sous-Stations équipées au moyen dé groupes moteur synchrone-dynamos.}

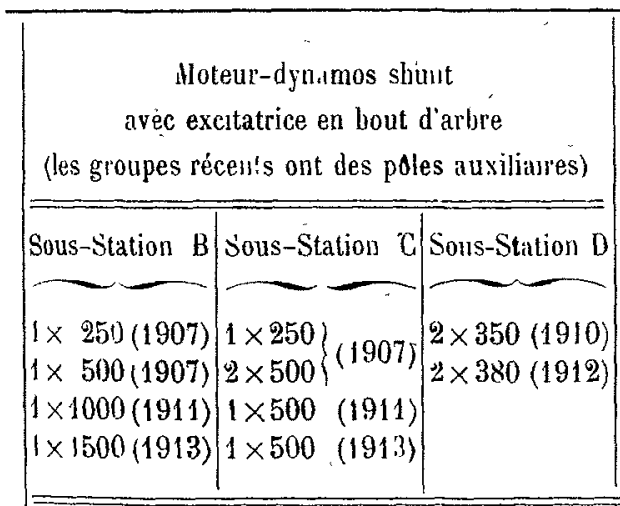

Moteur-dynamos, shunt

$31 \times 550 \ldots \ldots \ldots$

$6 \times 600 \ldots \ldots \ldots$

(1900)

$18 \times 1000 \ldots \ldots \ldots \ldots\left(1906-1^{20}\right)$

T'ous les group.s sauf ceux de 1900 sont

a poles atuxiliaires:

L'excitation des moteurs est fatte sous $2 \times 220 \mathrm{~V}$.
1 Les groupes synchrones onl été choisss parce qu'lls permettent de rendre le cos ô égal à 1 et d'assurer l'indépendance complète du côté continu et du côté alternatif. A l'époque de l'établissement du réstau, les constructeurs ne pouvaient pas garantir le fonction nement régulier des commutatrices à 50 périodes. A l'avenir on commandera de préférence des commutatrices à cause de leur rendement élevé et de leur marche silencieuse.

II. Temps requis pour la mise en charge d'un groupe $=$ environ $10^{\prime}$.

Chaque sous-station comporte une batterie-tampon munie d'un survolteur Prran.

L'exploitant apprécle beaucoup les avantages résultant de la suppression des transformateurs statiques: disposition plus simple et réduction des frais de premier établissement et de l'encombrement.

Afin d'êviter le bruit, trois groupes sont constitués de machines complètement fermées, refroidies par ventilation forcée.

Accidents survenus : 10 quelques bobines à HT endommagées au débu de l'exploitation ; 20 quelques rares décrochages à la suite de court-circuits.

Durée annuelle de fonctionnement d'un groupe : au maximum 6000 heures.
Hoteur-dynamos shunt

$2 \times 110 \ldots \ldots \ldots \ldots \ldots \ldots$

$1 \times 80 \ldots \ldots \ldots \ldots \ldots$

Moteur-dynano compound avec pôles auxiliaires

$1 \times 400 \ldots \ldots \ldots \ldots \ldots \ldots \ldots \ldots \ldots$

I. On a choisi les groupes synchrones afin d'obtenir un facteur de puissance convenable el de pouvoir élever la tension du réseau de traction jusqu'à concurrence de 600 volts au fur et à mesure que la charge augmente. D'autre part, les groupes du service de traction peuvent servir comme groupes de réserve pour le résean d'éclairage it 450 volts et inversement.

38.650 II. Temps nécessarre pour la mise en charge d'un groupe : environ 1.

Dans toutes les sous-stations les groupes de traction travaillent en parallèle avec une batterie-tampon sans survolteur. (Ces batteries n'mterviennent pas aux moments oil le diagramme de la charge accuse des pointes).

Une partie du réseau de traction est à 3 fils depuis le commencement de cette année.

Accidents survenus : Percement de l'isolant du stator contre la masse.

I. Les groupes synchrones ont êté adoptés d'une part afin d'améli rer le cos ọ du réseau général et d'autre part pour éviter les variations de tension.

II. Temps pour la mise en charge d'un groupe : $3^{\prime} 1 / 2$ pour $110 \mathrm{kw}, 5$ a 8 pour $100 \mathrm{kw}$.

Tous les groupes travaillent en parallèle avec une batterie tampon $\mathrm{m}$ nie d'un survolteur dont l'excitation shunt est alimentée à la tensıon de 300 volts (prise alternatıvement sur clacune des deux moitiés de la batterie).

Accidents survenus : Court-circuits au collecteur, dus a la poussièry qui s'étaít introduite entre les barres radiales parlant des lames, endroit difficilement accessible. Décrochages lors de grandes variations de la tension du côté alternatif ou de forts court-circuits du cóté contınu; les décrochares dus à cette dernière cause ont pu ètré évités par un réglage précis des déclanchements dutomatiquees des disjoncteurs.

Pas de réparations importantes.

Durée annuelle de service d'un groupe : environ 5.000 heures. 
TABLEAU XVI..- d) Résumé des réponses regues corcernant les Sous-Stations

\begin{tabular}{|c|c|c|c|c|c|c|c|c|c|c|c|}
\hline \multirow{3}{*}{ RÉSEAUX } & \multicolumn{4}{|c|}{ USLNE GENE'ATTHLE } & \multirow{3}{*}{$\begin{array}{l}\text { Services } \\
\text { réalisés }\end{array}$} & \multirow{3}{*}{ 总 } & \multirow{3}{*}{$\begin{array}{l}\text { Haute tension } \\
\text { alternative } \\
\text { voits }\end{array}$} & \multirow{3}{*}{$\begin{array}{l}\text { Tension } \\
\text { contisue } \\
\text { volls }\end{array}$} & & & \\
\hline & \multirow{2}{*}{$\begin{array}{c}\text { Puss.uce totale } \\
\text { lustallée } \\
\mathrm{kW}\end{array}$} & \multirow[b]{2}{*}{ Services réalisís } & \multirow{2}{*}{$\begin{array}{l}\text { Variation } \\
\text { de fréquence } \\
\text { en service }\end{array}$} & \multirow{2}{*}{$\operatorname{Cos} \rho$ moyen } & & & & & \multicolumn{3}{|c|}{$\begin{array}{c}\text { Conditions } \\
\text { dexploitation moyennes } \\
\text { sous } 10 \\
\end{array}$} \\
\hline & & & & & & & & & 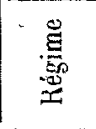 & 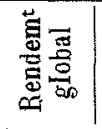 & $\begin{array}{l}0 \\
8\end{array}$ \\
\hline \multirow{2}{*}{$\begin{array}{c}\text { Tramways } \\
\text { municipaux de } \\
\text { Mannheim }\end{array}$} & \multirow{2}{*}{$\begin{array}{c}\text { HV }=2 \times 700 \\
1 \times 1400 \\
1 \times 2800 \\
1 \times 3000 \\
1 \times 300 \\
\Sigma=8900 \mathrm{lw}\end{array}$} & \multirow{2}{*}{$\begin{array}{c}\text { P moy }=2200 \mathrm{kw} \\
\text { dont } \\
\text { Tractıon }=500\end{array}$} & \multirow[t]{2}{*}{ Gnv. $1 \%$} & \multirow{2}{*}{ 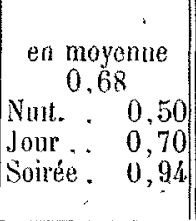 } & \multirow{2}{*}{ exclusivement } & \multirow[t]{2}{*}{50} & \multirow{2}{*}{$\begin{array}{r}4000 \\
\pm 50\end{array}$} & \multirow{2}{*}{$\begin{array}{c}550 \\
\dot{a} \\
600\end{array}$} & \multicolumn{3}{|c|}{ Groupe de $250 \mathrm{kw}$} \\
\hline & & & & & & & & & 0,92 & 0,88 & 1 \\
\hline \multirow{4}{*}{$\begin{array}{c}\text { Société } \\
\text { d'Eclairage } \\
\text { et de Transport } \\
\text { de force } \\
\text { Mendoza }\end{array}$} & \multirow{4}{*}{$\begin{array}{l}\text { TH }\left\{\begin{array}{l}1 \times 145 \\
1 \times 180 \\
2 \times 140 \\
2 \times 536\end{array}\right. \\
\text { HV }\left\{\begin{array}{l}2 \times 332 \\
1 \times 500\end{array}\right. \\
\Sigma=2841 \mathrm{kw}\end{array}$} & & \multirow{2}{*}{$\begin{array}{c}\begin{array}{c}\text { Garanties } \\
\text { pour } \mathrm{TH}\end{array} \\
\text { Variation }\end{array}$} & \multirow{4}{*}{$\begin{array}{c}\text { en moyenme } \\
0,84 \\
\text { varie entre } \\
0,92 \text { et } 0,75\end{array}$} & \multirow{4}{*}{$\begin{array}{l}\text { Traction } \\
\text { et } \\
\text { Eclarrage }\end{array}$} & \multirow[t]{4}{*}{50} & \multirow{4}{*}{$\begin{array}{c}4500 \\
\vdots \\
4900\end{array}$} & \multirow{4}{*}{$\begin{array}{c}440-550 \\
\text { pour traction } \\
.220=240 \\
\text { pour éclairage }\end{array}$} & 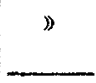 & 0,73 & $n$ \\
\hline & & & & & & & & & $4 / 4$ & 0,865 & 1 \\
\hline & & & clarge vicesse & & & & & & & & \\
\hline & & & \begin{tabular}{|r|r|}
$25 \%$ & $2 \%$ \\
$50 \%$ & $4 \%$ \\
$100 \%$ & $10 \%$ \\
\end{tabular} & & & & & & & & \\
\hline \multirow{5}{*}{$\begin{array}{c}\text { Chemins de Fer } \\
\text { Hessois } \\
\text { Darmstadt }\end{array}$} & \multirow[t]{4}{*}{$T V=2 \times 1600$} & \multirow{5}{*}{$\begin{array}{c}\text { Traction } \\
1 / 4 \text { à } 1 / 5 \\
\text { Eclairage } \\
\text { et } \\
\text { Force motrice } \\
3 / 4+4 / 5\end{array}$} & \multirow{5}{*}{$\begin{array}{c}\text { Négligeable } \\
\text { pour } \\
\text { variations } \\
\text { de charge } \\
\text { de } \\
15 \text { i } 20 \%\end{array}$} & \multirow{5}{*}{$\begin{array}{l}0,85 \\
\text { ùa } \\
\dot{0}, 89\end{array}$} & \multirow{5}{*}{$\begin{array}{c}\text { Traction } \\
\text { Eclairage } \\
\text { et } \\
\text { Force molrice }\end{array}$} & \multirow[t]{5}{*}{50} & & 550 & Grou & e de 30 & $\mathrm{kw}$ \\
\hline & & & & & & & -̀̀ & à & 0,66 & 0,76 & 0,90 \\
\hline & & & & & & & & 600 & $\begin{array}{l}4 / 4 \\
2 / 4\end{array}$ & $\begin{array}{l}0.87 \\
0,83\end{array}$ & 1 \\
\hline & & & & & & & & & Groll & e de 75 & $\mathrm{kw}$ \\
\hline & $\Sigma=3200 \mathrm{kw}$ & & & & & & & & $\begin{array}{l}4 / 4 \\
2 / 4\end{array}$ & $\begin{array}{l}0,91 \\
0,85\end{array}$ & $\begin{array}{l}1 \\
1\end{array}$ \\
\hline
\end{tabular}

TABLEAU XVI. - e) Résumé des réponses reques concernant les

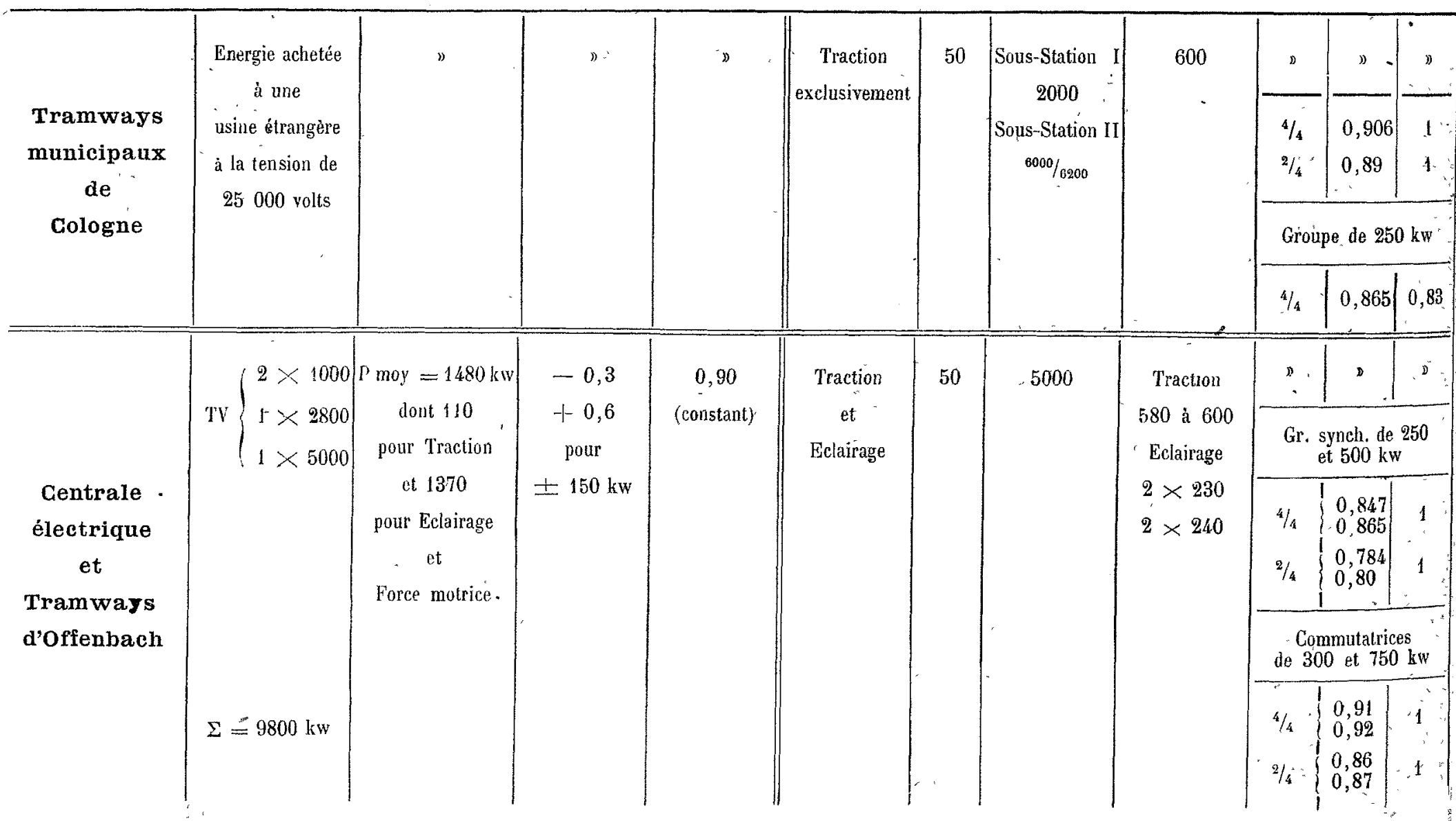




\begin{tabular}{|c|c|c|}
\hline $\begin{array}{c}\text { Nature et Puissance (en Kiws } \\
\text { des } \\
\text { Groupes de Transformation installés }\end{array}$ & $\begin{array}{l}\text { Puissance } \\
\text { totale } \\
\mathrm{Kw}\end{array}$ & $\begin{array}{l}\text { 1. Observations relatives à la justufication des systèmes employés. } \\
\text { II Observations diverses. }\end{array}$ \\
\hline 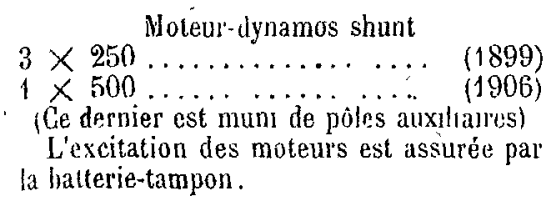 & $1.250^{\circ}$ & $\begin{array}{l}\text { I. Néant. } \\
\text { II. Durée de la mise en charge d'un groupe : 4' pour } 250 \mathrm{kw}, T \text { pour } 500 \mathrm{kw} \text {. } \\
\text { Accidents s'rvenus : décrochages a la suite de courl-crrcuits ou de fortes variations de charge. } \\
\text { L'induit d'un des groupes de } 250 \mathrm{kw} \text { a dì ètre remplacé aprés } 9 \text { ans de service. } \\
\text { Duréc annuelle de fonctionnement d'un groupe : } 3.040 \text { à } 4.400 \text { heures }\end{array}$ \\
\hline $\begin{array}{l}\text { Moteur-dynamos shiut } \\
\text { Traction : } 2 \times 250 \ldots \ldots \ldots \text { (1912) } \\
\text { (Dynanos avec pôles auxilaires) } \\
\text { Eclairage } 11 \times 90+2 \times 150 . \quad(1904) \\
\text { (Tous les moteurs ont des amortisseurs : } \\
5 \text { barres par pole). } \\
\text { Les moteurs sont excltés a } 220 \mathrm{~V} \text { par Ia } \\
\text { batterie d'éclairage }\end{array}$ & 890 & 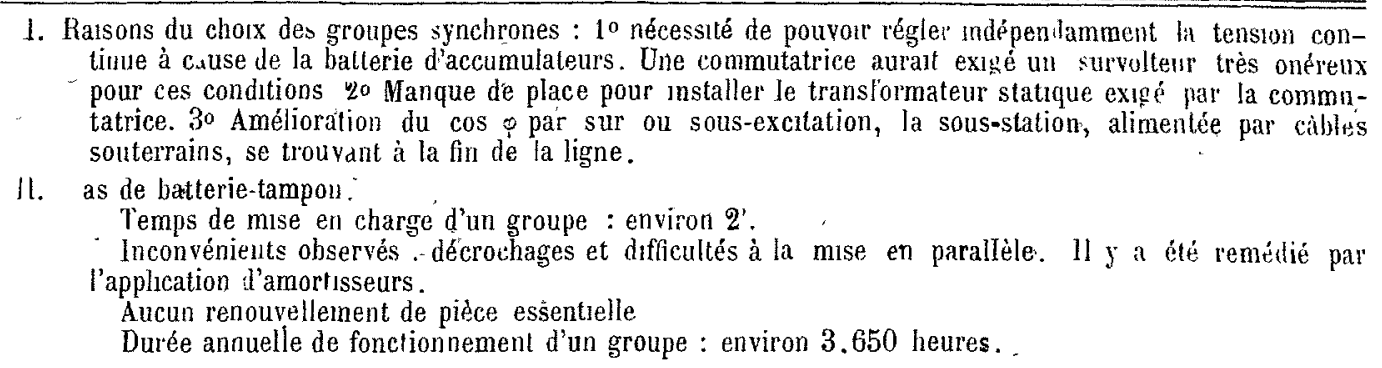 \\
\hline $\begin{array}{l}\text { Moteur-dynamos shunt à pỏles auxiliaires. } \\
1 \text { groupe de } 300 \mathrm{kw} \text { avec } 2 \text { dynamos dont } \\
\text { une à } 220 \mathrm{~V} \text { et l'autre à } 550 \mathrm{~V} . \text { (1911) } \\
2 \text { groupes de } 750 \mathrm{kw} \text { avec } 2 \text { dynamos à } \\
220 \mathrm{~V}-330 \mathrm{~V} \ldots \ldots \ldots \ldots \ldots \ldots \text { (1909) } \\
\text { Les moteurs sont excités à } 220 \mathrm{~V} \\
\text { par la batterie d'éclairage. }\end{array}$ & 1800 & 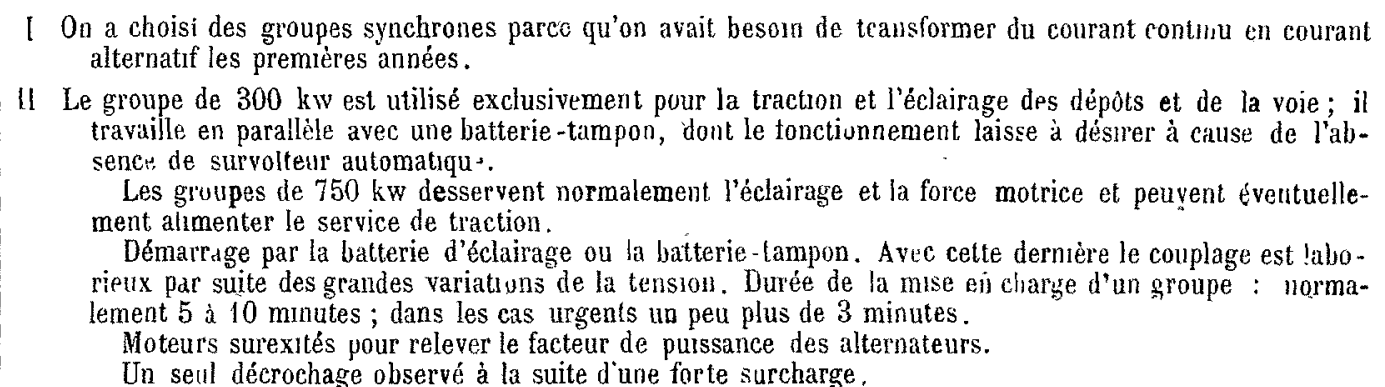 \\
\hline
\end{tabular}

\section{Sous-Stations dont l'équipement comprend plusieurs systèmes.}

Noteur synch-dynamos à pôles auxiliaires (3 paliers)

$4 \times 600 \ldots \ldots \ldots \ldots \ldots \ldots, \quad(1901)$

$1 \times 1000 \ldots \ldots \ldots \ldots \ldots \ldots$ (id. $)$

$2 \times 1000 \ldots \ldots \ldots \ldots \ldots \ldots \ldots(1909)$

(L'excitation des moteurs est taite à. 600 volts).

Moteur as nch-dynamos shunt

$1 \times 250 \ldots \ldots \ldots \ldots \ldots$
I La vitesse des moteurs synchrones étant constante pour toutes les charges, ll en résulte une tension fixe du coté continu.

L'excitation ne peut être réglée pour un cos p favorable

Le groupe asynchrone a été prévu à la fois pour permettre le démarrage des autres groupes en cas d'accident à la batterıe et pour alimenter le service de nuit.

II 'Temps de mise en charge d'un groupe : 2 ' $1 / 2$ à 3 '.

Les stators sont branchés sur le réseau par l'ntermédenre de bobines de self pour les moleur: syn. chrones $(2.000 \mathrm{~V}$ et $6.000 \mathrm{~V}$ ) et de résistances de choe, placées dans linterrupteur, pour le moteur asynchrone $(6.000 \mathrm{~V})$.

Dans les deux sous-stations les groupem suts lonctionnent en parallèle avéc une balterie-tampon par lintermédlarre d'un survolteur Prrani avec excitatrice en bout d'arhre dont l'excitation $\dot{a}$ fil fin est prise aux barres ommbus et l'enroulement série bronehé aux bornes d'un shunt intercalé du côté négatrs. Lil chute de tension fles dyıamos des groupes, de zéro à pleme charge, est d'envron $5 \%$

Pas observé de pumpage pour les moteurs synchrones.
Moleur synch-dynamos shunt à pòles auxiluaires, $(3$ puliers $)$

$1 \times 250$ (Iraction) $\ldots \ldots \quad(1909-10)$

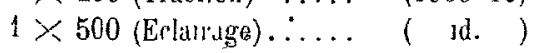

(Moteurs excités à 230 volts — Pas d'amortisseurs).

Commutatrices shuut av. survolteur alternatif (mumes d'amortusseurs en cage d'écureul)

$1 \times 300$ (Traction)........., (19/2)

$1 \times 750$ (Eclarrage)........ (id.)

(Tous ces groupes sont installés dans la Centrale).
I. Lors de la tondation de l'usihe (1909-10), on a choisi les groupes synchrones parce que les autres systèmes ll'avalent pas encore été suffisamment expérimentés dans la pratıqué.

Dans les extensions on a préléré adopter les commutatrices à cause de leur l'endement plus élevé et de leur mondre eticombi èment.

750 I[. Démarrage des commutatrices et des groupes du colé continu par la batterie d'éclairage de préférence. (Le synchrounsme est trés difficile à réaliser quand le démarrago est fatt par le courant des harres du servicę de traction).

'Temps de mise en charge : 3' à 4' pour les groupes; 2' à 3' pour lies commutatrices.

Tous les groupes de traclion fonctionnent e' parallète avec une batteric tampon mune d'an survolleur Prans.

Un troupe compensateur ade la hattere d'écharare à égahser les tensious sur les denx ponts.

Accidents survenus: In percement de lisolement dun stator. Lors de surcharges brusques, traîleces d'étincelles autour du collecteur, favorisées par un isolement insuffisant de ce derner par rapport à la masse.

Ni décrochage, no effets de pompage n'out élé observés pout aucune aachine (nu inversions de polarité pour les commutatrices)

Pas de renowvellement de pièce importante.

De zéro à pleine cliarge la tension des commutatric s varie de 600 a 590 volts. Le survolteur alternatif que chacune d'elles porte en bout d’arbre permet de regler la lension de - $5 \%$ jusque

t $10 \%$ pour l'éclainge it de $-20 \%$ jusque $+10 \%$ pour la fraction.

Durée annuelle de foncliomsement d'une machine : Truction : 5.800 el 6.300 hemres. Eclainage : 700 et 3.600 heures. 
TABLEAU XVI. - e) Résumé des réponses regues concernant les Sous-Stations

\begin{tabular}{|c|c|c|c|c|c|c|c|c|c|c|c|}
\hline \multirow{3}{*}{ RESEAUX } & \multicolumn{4}{|c|}{ USINE GÉNÉRATRICF } & & & & \\
\hline & \multirow{2}{*}{$\begin{array}{c}\text { Puissance totale } \\
\text { iustallée } \\
\mathrm{KW}\end{array}$} & \multirow{2}{*}{ Services réalisés } & \multirow{2}{*}{$\begin{array}{c}\text { Variation } \\
\text { de fréquence } \\
\text { en service }\end{array}$} & \multirow{2}{*}{$\operatorname{Cos} \varphi$ moyen, } & \multirow{2}{*}{$\begin{array}{l}\text { Services } \\
\text { Iéalisés }\end{array}$} & \multirow{2}{*}{ 总 } & \multirow{2}{*}{$\begin{array}{l}\text { Haute tension } \\
\text { alternative } \\
\overline{v_{0}} \text { 'ts }\end{array}$} & \multirow{2}{*}{$\begin{array}{c}\text { Tension } \\
\text { contiıue } \\
\text { volts }\end{array}$} & \multicolumn{3}{|c|}{$\begin{array}{c}\text { Conditions } \\
\text { d'exploitation moyennes } \\
\text { sous 10 } \\
\end{array}$} \\
\hline & & & & & & & & & 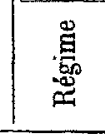 & 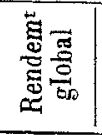 & $\begin{array}{l}0 \\
\text { है }\end{array}$ \\
\hline \multirow{7}{*}{$\begin{array}{c}\text { Centrale } \\
\text { électrique } \\
\text { de } \\
\text { Dresde }\end{array}$} & \multirow{7}{*}{$\begin{array}{c}\mathrm{TV}=2 \times 6500 \\
\mathrm{MV}\left\{\begin{array}{rrr}2 \times 750 \\
2 \times & 650 \\
4 & \times & 450 \\
\Sigma & =17600 \mathrm{kw}\end{array}\right. \\
\text { En plus } \\
5 \times 800 \mathrm{kw} \text { ent } \\
\text { MV génér. } \\
\text { couraut continu }\end{array}$} & \multirow{7}{*}{$\begin{array}{c}\text { Traction } \\
\text { Eclanrage et } \\
\text { Transport de Force } \\
\text { (courant contin }) \\
5000 \mathrm{kw} \\
\text { Eclairage et } \\
\text { Transport de Force } \\
\text { (courant alternatif } \\
6000 \mathrm{kw}\end{array}$} & \multirow{7}{*}{$\begin{array}{c}\text { au max. } \\
5 \% \\
\text { pour variation } \\
\text { de charge } \\
\text { d. } 50 \%\end{array}$} & \multirow{7}{*}{$\left|\begin{array}{c}\text { Variable } \\
\text { entre } \\
0,56 \text { et } 0,87\end{array}\right|$} & \multirow{7}{*}{$\begin{array}{c}\text { Traction } \\
\text { principalement } \\
\text { mais auss } \\
\text { Eclairage et } \\
\text { Transport de } \\
\text { Force }\end{array}$} & \multirow[t]{7}{*}{50} & \multirow{7}{*}{10.000} & \multirow{7}{*}{$\begin{array}{c}520 \\
\dot{\mathfrak{a}} \\
540\end{array}$} & $"$ & D & $n$ \\
\hline & & & & & & & & & Grou & dé 100 & $0 \mathrm{kw}$ \\
\hline & & & & & & & & & $\frac{1 / 4}{2 / 4}$ & $\begin{array}{l}0,865 \\
0,82\end{array} \mid$ & $\begin{array}{l}1 \\
1\end{array}$ \\
\hline & & & & & & & & & Grou & es de 75 & $0 \mathrm{kw}$ \\
\hline & & & & & & & & & $\begin{array}{l}4 / 4 \\
2 / 4 \\
\end{array}$ & $\begin{array}{l}0,855 \\
0,80\end{array}$ & $\begin{array}{l}1 \\
1 \\
\end{array}$ \\
\hline & & & & & & & & & Woteur & générr de & $250 \mathrm{kn}$ \\
\hline & & & & & & & & & $\begin{array}{l}4 / 4 \\
2 / 4 \\
\end{array}$ & $\begin{array}{l}0,815 \\
0,795 \\
\end{array}$ & $"$ \\
\hline \multirow{5}{*}{$\begin{array}{c}\text { Tramways } \\
\text { municipaux } \\
\text { de } \\
\text { Zurich }\end{array}$} & \multirow{5}{*}{$\begin{array}{c}\text { E.tergle fournie } \\
\text { par } \\
\text { I'Usine "Albuld " } \\
\text { de la } \\
\text { Vulle de Zurich } \\
\text { TH } \\
M V\end{array}$} & \multirow{5}{*}{$\begin{array}{c}\text { Traction } \\
2800 \mathrm{kw} \\
\text { Eclairage } \\
4000 \mathrm{kw} \\
\text { Trausport de Force } \\
11800 \mathrm{kw}\end{array}$} & \multirow{5}{*}{$\begin{array}{c}\text { Lors de } \\
\text { décharges } \\
\text { importautes : } \\
5 \% / \% \text { pour } \mathrm{TH} \\
3 \% \text { pour } \mathrm{MV}\end{array}$} & \multirow{5}{*}{$\begin{array}{c}\text { Force } \\
0,8 \text { à } 0,85 \\
\text { Eclaurage } \\
1\end{array}$} & \multirow{5}{*}{$\begin{array}{c}\text { Traction } \\
\text { exclusivement }\end{array}$} & \multirow[t]{5}{*}{50} & \multirow{5}{*}{$\begin{array}{l}5.700 \\
\stackrel{a}{ } \\
6.300\end{array}$} & \multirow{5}{*}{$\begin{array}{c}580 \\
\grave{a} \\
600\end{array}$} & $n$ & $D$ & 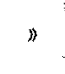 \\
\hline & & & & & & & & & Gr. A & $\begin{array}{l}\text { synch de } \\
700 \mathrm{kw}\end{array}$ & 500 \\
\hline & & & & & & & & & $\begin{array}{l}4 / 4 \\
2 / 4\end{array}$ & $\begin{array}{l}0,85 \\
0,78\end{array}$ & $\begin{array}{l}D \\
D\end{array}$ \\
\hline & & & & & & & & & Gr. Asy & ch. de 7 & $00 \mathrm{kw}$ \\
\hline & & & & & & & & & $\begin{array}{l}4 / 4 \\
2 / 4\end{array}$ & $\begin{array}{l}0,90 \\
0,80\end{array}$ & $\begin{array}{l}1 \\
1\end{array}$ \\
\hline \multirow{9}{*}{$\begin{array}{c}\text { Centrale } \\
\text { électrique } \\
\text { de } \\
\text { Munich }\end{array}$} & Diffërentes & P moy. $=4080 \mathrm{kw}$ & $\mathrm{THI}= \pm 2 \%$ & Ser,siblement & Traction & 50 & 5.000 & 650 & " & ") & 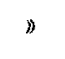 \\
\hline & $\left|\begin{array}{c}\text { usines comportan } \\
\text { ensemble }\end{array}\right|$ & Traction $1 \times 90 \mathrm{kw}$ & $M V=-25 \%$ & & & & & & Grour & es synch & rones: \\
\hline & $\begin{array}{c}\text { 14 alternateurs } \\
\text { de } \\
1400,890,800\end{array}$ & $\begin{array}{c}\text { et } \\
\text { Eclairage et Force } \\
2190 \mathrm{kw}\end{array}$ & & & $\begin{array}{c}\text { par } \\
\text { arcs branchés } \\
\text { sur la } \\
\text { tension totale }\end{array}$ & & & 700 & $\begin{array}{l}4 / 4 \\
2 / 4 \\
1 / 4\end{array}$ & $\begin{array}{l}0,875 \\
0,825 \\
0,71\end{array}$ & $\begin{array}{l}1 \\
1 \\
1\end{array}$ \\
\hline & $\begin{array}{c}\text { à } 700 \mathrm{kw} \\
\text { mus par MV et TH }\end{array}$ & & & & & & & & Conve & trs en ca & scadt \\
\hline & 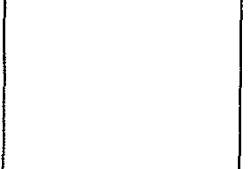 & & & & & & & & $\begin{array}{l}4 / 4 \\
2 / 4 \\
1 / 4\end{array}$ & $\begin{array}{l}0,912 \\
0,898 \\
0,84\end{array}$ & $\begin{array}{r}1 \\
0,99 \\
0,98\end{array}$ \\
\hline & $\Sigma=13.070 \mathrm{kw}$ & & & & & & & & Comr & $\begin{array}{l}\text { utatrice : } \\
\text { et Régul }\end{array}$ & $\begin{array}{l}\text { avec } \\
\text { ateur }\end{array}$ \\
\hline & & & & & & & & & $\begin{array}{l}4 / 4 \\
2 / 4 \\
1 / 4\end{array}$ & $\begin{array}{l}0,945 \\
0,925 \\
0,877\end{array}$ & $\begin{array}{l}1 \\
1 \\
1\end{array}$ \\
\hline & & & & & & & & & $\begin{array}{l}\text { Tous } \\
\text { sont dé } \\
\text { des pe }\end{array}$ & $\begin{array}{l}\text { es renden } \\
\text { erminús. } \\
\text { nétliode } \\
\text { ttes sépar }\end{array}$ & $\begin{array}{l}\text { nents } \\
\text { par' } \\
\text { ées.: }\end{array}$ \\
\hline & & & & & & & & & & & \\
\hline
\end{tabular}


dont l'équipement comprend plusieurs systemes (súite).

SOUS-STATIONS DE TRANSFORMATION

\begin{tabular}{|c|c|c|}
\hline $\begin{array}{l}\text { Nature et Puissance (en Kw) } \\
\text { des } \\
\text { Groupes de Transformation installés }\end{array}$ & $\begin{array}{c}\text { Puissancer } \\
\text { totale } \\
\text { par réseau } \\
\mathrm{Kw}\end{array}$ & $\begin{array}{l}\text { I. Observations relatives à ld justification des systèmes emplojés. } \\
\text { II Observations diverses. }\end{array}$ \\
\hline
\end{tabular}

Moteur syuch-dynamos shunt à pôles auxili ures (3 palıers)

$1 \times 1000 \ldots \ldots \ldots \ldots \ldots,\{1912)$

$2 \times 750 \ldots \ldots \ldots \ldots \ldots \ldots(1912)$

$2 \times 750$ (élablıs à la Centrale st alımentés

i 2000 volts) . ...............

(L'excitation dos moteurs est faite à $110 \mathrm{~V}$ )

Hoteur asynch-dynanıo shunt à pôles auxilaires avec transf, -statique $10000 \mathrm{v} / 500 \mathrm{v}$

$1 \times 250$

Moteur asynch-dynamos shunt a pòles auxiliaires ( 4 paliers)

12 groupes de 300,500 et $700 \mathrm{kw}$ (1898.1913)

Joteur synch-dynamos shunt à pôles auxiliarres ( 4 paliers)

$1 \times 700$.

(Excitalrice à $60 \mathrm{~V}$ en bout d'arbre).
I. Les groupes «synchrones » ont été choisss pour rolever le facteur de punssance du réseau génėral et le groupe «asynchrone p pour permettre de démarrer du côté alternatif dans le cas où le courant continu feralt défaut.

II. Démarrage du groupe ssynchrone avec plase auxliaire, ré:istance et bobine de self; temps requis : environ 1'. Temps de mise en marche des groupes "synchrones ": 3 ' à 4'.

4.000

Pas de batterie-tampon. Tous le: groupes marchent en parallèle avec les génératrices actionnées par les machnes à vapeur de la centrale.

L'exploutant n'indique pas comment est réalicée la division de la tension pour le réseau de distribution à deux ponts du service d'éclairage.

Il n'a été observé ni décrochages, ni phénomènes de pompage pour les moteurs synchrones Durée annuelle de fnnctionnement d'un groupe -3.000 à 5.000 heures.

250

I Les groupes « asynchrones ont été imposés par la ville, qui fournit le courant. Le groupe a synchrone 》a été instalé pour relever le lacteur de puissance.

Malgré la stabilité plus grande des moteurs asynchrones, l'exploitant donnerait maintenant la préférence à des groupes a synchrones $\$$ de grande puissance.

II. Démurrage des moteurs asynchrones par rhéostat métallique ou par rhéoutat liquite (démarrage plus doux), avec $30^{\circ} \%$ du courant normal. Temps de mise en charge $2{ }^{\prime}$ a $3^{\prime}$ Application brusque de la tension totale au stator ; il ne s'est $j$ tmas produit de claquage .

Temps nécessaire pour la mise en charge du groupe syuchrone : environ $5^{5}$.

Tous les groupes fonclionnent en parallèle avec une batterie-tampon sans survolteur; Ja tension aux barres varie de 5 à $10 \%$ suivant la charge.

Accidents survenus : court-circuits dans le rotor des moteurs asynchrones dus ì l'assemblage défectueux des barres

Durée annuelle de fonctionnement d'un groupe 4.000 à $5 \quad 00$ heures.
Moteur synch dynamos slunt á pótes auxılianres (3 paliers Pas d'amortisseurs).

$2 \times 700$.

$1 \times 790$

$1 \times 360$

$2 \times 800$.

(Exctution des moleurs à 220-230 $\mathrm{V}$ au moyen d'une latterte).

Convertsseur en carcade à excitation shunt at pôles auxiluare's 12 praliers. Amortisseur en cage d'icureul 8 burres pur pole).

$2 \times 800$.

$2 \times 900$

$2 \times 1000 \ldots$

Commutatrices hexaplasées shunt à pìles auxilaires avec denx collecteurs et un régulatcur de potentirl

$1 \times 1000 \ldots \ldots \ldots \ldots$

(Amortisseurs constitués par 3 barres par póth).
I. On u’avait le choix, à l'origine, qu'entre les groupes moteurs-généra'eurs " synchrone " et " asynchrone $n$. Les premiers ont été choisis à cause de leur bon facteur de pussance et de leur faculté de produire du courant triphąsé en cas d'accident aux altermateurs ou à la lig»le d'alimentation.

A partir de 1910 on a prétéré les coivertisseurs en caścale aux moteurs générateurs, à catuse de leur rendement plus élevé, de leur focluté de démarrage de leur meilleure commutation et de la plus grande sécurté de service qui résulte de leur vites`e réduite.

(Au point de vue du prix. l'exploitant fait la renarque suivante : lors d'une soumission en 1910, les convertisseurs eu cascade cuutaient de $7 \%$ à $35 \%$ plus cher que les gruupes moteurs-générateurs. Ces deruiers étaient au mème prix que les commutatric s simples, transformatenrs compris).

La commutatrice simple a été installée à còté des convettisstur's en cascade afin de pouvorr comparer en service ces deux systèmes de machines.

II. Démarrage des groupes synchrou'es du côté contınu par 2 transformateurs rolatıfs en série branchés sur la réseau. Durée du enuplage normalteme' $\mathrm{t}$ '2' à 3 ', sl fréquence 't tension varjent. 10 ' et mème davantage.

Temps nécessué par la mise en charge d un convertisseur en cascade : au maximum 2' à 3'.

Démarrage de la commutatrice par moteur de lancement. Temps requis : 2' à 3' quand la tension et la périodirité ne iarient pas.

L s groupes synchrones of les convertsseurs ell ciscade travallent directement en parallèle avec une latterie-tampon (La chute de tension de zéro à pleine charg, á excitation shunt fixe, est de $13 \mathrm{o} / \mathrm{n}$ pour les groupes s ynchrones et de $6,5 \%$ pour les convirlisseurs)

Valeurs du cos o des convertisseurs en ascade pour différentes charges et différentes tensions: :

La commutatrice comporte, entre son induit et son transformateur, un régulateur de potentiel capable, (suivent le rapport de

\begin{tabular}{c|c|c|c|c}
\hline Ten-ion & $4 / 4$ & $3 / 4$ & $2 / 4$ & $1 / 4$ \\
\hline 680 & 1 & 1 & 0,99 & 0,98 \\
700 & 1 & 0,99 & 0,97 & 0,94 \\
790 & 0,99 & 0,96 & 0,93 & 0,86
\end{tabular}
transformation ulilisé $>$, de faire varier la

terrsion de $636 \mathrm{~V}$ a $709 \mathrm{~V}$ ou de $656 \mathrm{~V}$ à $730 \mathrm{~V}$ Ce régulateur abaisse le rendement global de $0,75 \%$ à plene charge ef de $1,5 \%$ à $1 / 4$ de charge

La commutatrice est aưsi employée comme machine de réserve pour le réseau à basse tension de $2 \times$ $110 \mathrm{~V}$ et $2 \times 220 \mathrm{~V}$. Elle a d'autre part été déjà utilıs ${ }^{2}$ à rebours sans aucun inconvénient.

Accide its survenus :

10 Aux groupes synchrones: Percement de l'isolement de l'inducteur Percement de l'isolement du stator à 3 reprises différentes

2o Converisseurs en cascade : Etincelles autour du collecteur déterminées par un défaut d'isolement de ce dernier.

3o Commutatrice : Rupture de bandages au collecteur.

Pour aucune machine il n’a été obsı rvé ui décrochage, ni pompage (ni inversion de polarité pour la commutatrice)

L'explotant estime que les frais d'entretien annuels sont sensiblement les mêmes pour les groupes des 3 systèmes.

Durée annuelle de fonctionnement d'un groupe : en général de 5.000 à 7.000 heures. 